\title{
MUJERES Y MEMORIA EN LA CIUDAD DE MADRID
}

\author{
WOMEN AND MEMORY IN THE CITY OF MADRID
}

\author{
Almendra Aladro *, Lucía Escalante **
}

\begin{abstract}
RESUMEN: A partir del cruce de los resultados de dos proyectos de investigación financiados por SEGIB y Fundación Carolina decidimos analizar el debate suscitado ante el intento de eliminar el mural feminista de Ciudad Lineal (Madrid) fogoneado por el partido Vox como una continuidad de las políticas en materia de espacios de memoria histórica y dentro de los debates feministas sobre el acceso y goce del derecho a la ciudad. Identificamos las categorías analíticas necesarias para fundamentar el análisis de discurso al que sometimos tanto al material periodístico recolectado como a las actas de los debates legislativos sobre la cuestión. Concluimos que esta disputa trasciende una decisión estética o urbanística en sentido estricto. En ella subyace la lucha por la toma de decisiones en la ciudad en general, y por quiénes logran inscribir la memoria en el espacio en particular. La escasez de espacios de la memoria que satisfagan los estándares internacionales en la materia perpetúa las inscripciones que invisibilizan a las víctimas del franquismo y antecede y fundamenta la exclusión de las mujeres del espacio público.
\end{abstract}

ABSTRACT: From the intersection of the results of two research projects financed by SEGIB and Fundación Carolina, we decided to analyze the debate raised by the attempt to eliminate the feminist mural of Ciudad Lineal (Madrid) fired by the Vox party as a continuity of policies regarding spaces of historical memory and within feminist debates on access to and enjoyment of the right to the city. We identified the analytical categories necessary to base the discourse analysis 1 to which we submitted both the journalistic material collected and the minutes of the legislative debates on the issue. We concluded that this dispute transcends an aesthetic or urban decision in the strict sense. Underlying it is the struggle for decision-making in the city in general, and for those who manage to inscribe memory in space in particular. The scarcity of memory spaces that meet international standards on the matter perpetuates the inscriptions that make the victims of the Franco regime invisible and precedes and grounds the exclusion of women from public space.

PALABRAS CLAVE: derecho a la ciudad, mujeres, género, memoria.

KEYWORDS: right to the city, women, gender, memory.

Fecha de recepción: 01/11/2021

Fecha de aceptación: 01/11/2021

doi: https://doi.org/10.20318/universitas.2022.6587 * Abogada. Becaria doctoral (CONICET) en el CIC-CIDDH-UNMDP. Ex Becaria de
Estancias Cortas (SEGIB-FC) en el IDHPB (UC3M). Docente de la cátedra "Derecho
I" de la carrera de Trabajo Social (UNMDP). E-mail: aaladro@mdp.edu.ar
** Abogada. Becaria doctoral (CIN, Programa PERHID). Docente de la asignatura
"Derecho Político" de la carrera de Abogacía (UNMDP) e integrante del grupo de
investigación "Pensamiento Crítico" (CIC-CIDDH-UNMDP). Ex Becaria de Estancias
Cortas (SEGIB-FC) en el IDHPB (UC3M). E-mail: lucia escalante@hotmail.com 


\section{1.-INTRODUCCIÓN}

En enero de 2021 el partido VOX inició una cruzada contra la representación de las mujeres en el espacio público madrileño que fue acompañada por el conservadurismo local con representación en los aparatos gubernamentales de toma de decisiones. La acometida fue contra el popularmente conocido como "mural feminista de Ciudad Lineal", una obra callejera de 60 metros de longitud emplazada en el Polideportivo de la Concepción en el año 2018 en el marco del proyecto "Compartiendo Muros" del Ayuntamiento. Esta disputa motiva el análisis que aquí se presenta y que pretende indagar en el uso del espacio público en clave de géneros y memoria como parte de un debate político aún mayor respecto del acceso y el goce del derecho a la ciudad.

El presupuesto de partida sostiene que el espacio contiene materialmente a la memoria, memoria inscrita que, a su vez, es una herramienta estatal para cumplir con la carga de garantizar su recuperación en términos de responsabilidad internacional con los derechos humanos. En este orden de ideas, es central la postura de Karen Till respecto de la inscripción espacial de la memoria para la visibilización y accesibilidad universal y la configuración de un derecho a representar el pasado dentro de procesos más amplios de democratización ${ }^{1}$.

Dentro de la exclusión de las mujeres del acceso y goce del derecho a la ciudad ${ }^{2}$ afirmamos aquí que la inscripción de las memorias de las mujeres es otro derecho negado. La justicia transicional incorporó en los últimos años un enfoque que pretende develar los mecanismos de opresión de género en miras a las denuncias más profundas sobre las desigualdades. Se consideran clave en este punto a los aportes sobre experiencia y memoria ${ }^{3}$ y la potencia de la resignificación ${ }^{4}$, como así también respecto de la movilización de la memoria como política activa contrahegemónica frente a los movimientos reaccionarios y neoconservadores ${ }^{5}$.

\footnotetext{
${ }^{1}$ Karen Till, 'Wounded cities: Memory-work and a place-based ethics of care' (2012) 31 Revista Political Geography 1.

2 Almendra Aladro, 'Capitalismo, patriarcado y ciudad: generalidades de la producción de lo urbano desde una perspectiva de género' (2019) 21 Revista Derecho y Ciencias Sociales; y Almendra Aladro 'El acceso a la ciudad de las mujeres' [2020], II Coloquio Internacional de Conflictos Urbanos, Jaén de la Frontera, Universidad de Cádiz.

${ }^{3}$ Lilian Elmira Celiberti, 'Desatar, desnudar... reanudar' (2015) 39 Revista Estudios de Sociología, Araraquara 291.

${ }^{4}$ Claudia Bacci, 'Ahora que estamos juntas: memorias, políticas y emociones feministas' (2020) 28 Revista Estudios Feministas 1.

5 Marianne Hirsch, 'Introduction: Practicing Feminism, Practicing Memory', en Ayşe Gül Altınay and María José Contreras, Marianne Hirsch, Jean Howard, Banu Karaca \& Alisa Solomon(Eds..) Women Mobilizing Memory (Columbia University Press 2019).
} 
La estrategia metodológica que desplegamos pretendió facilitar la indagación en las representaciones y estrategias de los actores y actoras que participaron en el conflicto alrededor del mural. En primer lugar, realizamos un profundo análisis de literatura especializada para identificar las categorías analíticas que, en segundo lugar, fundamentaron el análisis de discurso ${ }^{6}$ al que sometimos tanto al material periodístico recolectado como a las actas de los debates legislativos sobre la cuestión. Finalmente, se realizó una entrevista en profundidad semiestructurada y la informante se seleccionó mediante la técnica de muestra de casos sumamente importantes para el problema analizado? ${ }^{7}$.

\section{2.- CIUDAD, ESTUDIOS DE GÉNERO Y MEMORIA}

Esta reconstrucción teórica permite una comprensión translocal y multiescalar de la subjetivación histórica y política de las mujeres desde una perspectiva espacial que problematiza quién, cómo y dónde inscribe la memoria en las ciudades.

Maurice Halbwachs fue el primero en acuñar el concepto de memoria colectiva, su idea central es que además del proceso de retención vinculado con los procesos fisiológicos del cerebro de cada individuo (memoria individual), hay que suponer la existencia de una memoria social que preserva el saber sobre acontecimientos, valores y relaciones dentro de un grupo. Así, el concepto de memoria colectiva permite pensar la memoria y sus objetos en unidades sociales mayores.

El autor advirtió tempranamente la relación existente entre memoria y espacio al postular que la mayor parte de los grupos dibujan de alguna manera su forma en el suelo y encuentran sus recuerdos colectivos en un marco espacial definido de esta manera. El espacio es entonces junto con el tiempo uno de los marcos sociales de la memoria de la comunidad, que implican construcciones y representaciones colectivas de la identidad de cada grupo.

El espacio público de las ciudades es un ámbito propicio donde inscribir la memoria dado su visibilización y accesibilidad para toda la comunidad. La importancia de la construcción de la memoria democrática en el espacio cotidiano de la ciudadanía recae en que sirve como test sobre la democracia ${ }^{8}$.

Karen Till logró dar cuenta de la relación que existe entre la memoria y el derecho a la ciudad a través de los estudios etnográficos realizados en diferentes comunidades. La autora parte del concepto

\footnotetext{
6 Irene Vasilachis de Gialdino, Discurso Político y Prensa escrita. Un análisis sociológico, jurídico y lingüístico (Gedisa, 1997).

7 Roberto Hernández Sampieri, Carlos Fernández Collao y Pilar Baptista Lucio, Metodología de la investigación (McGraw-Hill Interamericana, 2006).

8 Jordi Borja, Revolución urbana y derechos ciudadanos (Café de las Ciudades, 2014). 
acuñado por Lefebvre de derecho a la ciudad y reconoce que los Estados democráticos suelen negar a sus habitantes el acceso equitativo a sus recursos, incluyendo entre estos el derecho a narrar el pasado a través de medios legales y materiales en el espacio urbano; concluye que este derecho a representar el pasado puede ser considerado como parte del derecho a la ciudad y está íntimamente ligado a los procesos de democratización ${ }^{9}$. Se torna clave para la complejización del cotidiano urbano, además, la escala de "lo barrial" como un ánima social de alta influencia en la subjetivación subalterna ${ }^{10}$ y se relaciona estrechamente con la identidad, categoría clave que mediatiza el vínculo entre género y memoria ${ }^{11}$.

La dicotomía entre público-privado revela en los estudios de género cómo las mujeres desde una visión esencialista han estado excluidas históricamente del espacio público y, por ende también de la posibilidad de inscribir sus memorias. En las últimas décadas, el feminismo ha reivindicado el rol de las mujeres en la apropiación de los espacios públicos que tradicionalmente le han sido negados ante la importancia de su presencia en la toma de decisiones sociales ${ }^{12}$, y estos debates han trascendido tanto al ámbito de los estudios urbanos como a los de memoria.

Afirma Hirsch ${ }^{13}$ que la incorporación del enfoque de género al estudio de la memoria ha sido más bien tardío, dando lugar desde una perspectiva crítica, a lo que se conoce como estudios feministas de la memoria basados en la práctica. Frente al avance de las derechas a nivel global, junto con la exaltación del nacionalismo, el etnocentrismo y el imaginario masculino, los estudios feministas de la memoria ofrecen una nueva lectura y tácticas de resistencia históricas ${ }^{14}$, mientras que ponen en valor el lugar de las mujeres en la producción de conocimiento y en el desarrollo de la historia ${ }^{15}$. Así, una comunión renovada entre género y memoria permite dar cuenta de la continuidad histórica entre la organización por los derechos humanos en contextos

\footnotetext{
9 Karen Till, 'Wounded cities: Memory-work and a place-based ethics of care' (2012)

31 Revista Political Geography 1.

10 José Ignacio Larreche, 'Lo barrial del barrio. Una discusión desde el habitar subalterno en Bahía Blanca (Argentina)' 15 Geograficando 69.

${ }^{11}$ Lelya Elena Troncoso Pérez \& Isabel Piper Shafir, 'Género y memoria:

articulaciones críticas feministas' (2015) 15 Revista Athenea Digital 65, 69.

12 Catharine Mackinnon, Feminism Unmodified: Discourse on Life and Law (Harvard University Press, 1987).

13 Marianne Hirsch, 'Introduction: Practicing Feminism, Practicing Memory', en Ayşe Gül Altınay and María José Contreras, Marianne Hirsch, Jean Howard, Banu Karaca \& Alisa Solomon(Eds..) Women Mobilizing Memory (Columbia University Press 2019). 14 Marianne Hirsch, 'Introduction: Practicing Feminism, Practicing Memory', en Ayşe Gül Altınay and María José Contreras, Marianne Hirsch, Jean Howard, Banu Karaca \& Alisa Solomon(Eds..) Women Mobilizing Memory (Columbia University Press, 2019).

15 María Cristina Ferrer González, 'Memoria histórica y democrática con perspectiva de género' (2019) 96 Revista PH, 218.
} 
de violencia estatal y la lucha contemporánea de las mujeres ${ }^{16}$. Esta colaboración recíproca entre ambos campos ha dado lugar tanto a la "memorización del género" como a la "generización de la memoria"17.

\section{3.- MATERIALES Y MÉTODOS}

La estrategia metodológica seleccionada es primordialmente etnográfica ${ }^{18}$ y está escalonada en dos momentos. El primero buscó caracterizar las acciones políticas de VOX en relación al mural como así también las respuestas de otros espacios políticos institucionalizados. Para esto realizamos un análisis de discurso ${ }^{19}$ que tuvo como materia prima las actas y documentos en las cuales constan intervenciones en el el Pleno del Distrito de Ciudad Lineal y el Pleno del Ayuntamiento de Madrid los días 21 y 29 de enero de 2021 respectivamente.

El segundo escalón pretendió reconstruir cronológicamente los hechos ocurridos en Ciudad Lineal poniendo énfasis en las estrategias de comunicación y acción tanto de los grupos políticos con representatividad política como de la comunidad local. Para ello se realizó un análisis de discurso ${ }^{20}$ que echó mano de las notas periodísticas y de opinión publicadas en El Público, diario digital independiente y autofinanciado comprometido con las poblaciones vulnerables, la situación de la mujer y una visión progresista de la memoria histórica (El Público, 2018) que aporta una mirada de los hechos ocurridos congruente con el marco teórico aquí adoptado. Se seleccionaron para el análisis notas periodísticas y de opinión publicadas entre el 21 y el 30 de enero de 2021 inclusive.

Por último, se realizó una entrevista en profundidad utilizando la técnica de muestra de casos sumamente importantes para el problema analizado ${ }^{21}$, en tanto la informante cuenta con una amplia trayectoria en las luchas feministas llevadas adelante en la ciudad de Madrid y más recientemente también en aquellas relacionadas a la memoria histórica, empecíficamente participó activamente en la organización de las movilizaciones vecinales en defensa del mural feminista de Ciudad Lineal.

\footnotetext{
16 Jelin, E. \& Sutton, B., 'Memoria, género y activismo. Resistencia a la dictadura y lucha por el aborto legal' (2021) 11 Aletheia.

17 Lelya Elena Troncoso Pérez \& Isabel Piper Shafir, 'Género y memoria:

articulaciones críticas feministas' (2015) 15 Revista Athenea Digital 65, 66.

${ }^{18}$ Aldo Ameigueiras, 'El abordaje etnográfico en la investigación social', en Irene Vasilachis de Gialdino (Coord.) Estrategias de investigación cualitativa (Editorial Gedisa, 2006) 107.

19 Daniel Balinhas, 'Populismo y nacionalismo en la "nueva" derecha radical española' (2020) 13 Pensamiento al margen. Revista Digital de Ideas Políticas 69.

20 Irene Vasilachis de Gialdino, Discurso Político y Prensa escrita. Un análisis sociológico, jurídico y lingüístico (Gedisa, 1997).

${ }^{21}$ Roberto Hernández Sampieri, Carlos Fernández Collao y Pilar Baptista Lucio, Metodología de la investigación (McGraw-Hill Interamericana, 2006).
} 


\section{4.-RESULTADOS}

El partido VOX cuestiona de manera sistemática las políticas de equidad de género. A nivel nacional, hacen uso de la facultad de presentación de preguntas contemplada en el artículo 185 y ss. del Reglamento del Congreso de los Diputados. Los focos de cuestionamiento son variados, pero siempre exigiendo al Poder Ejecutivo la justificación de las medidas de acción positiva en favor de las mujeres. Hemos encontrado cuestionamientos lanzados en relación a la relación entre poder y trabajo ${ }^{22}$, las políticas sociales destinadas a las mujeres en general con énfasis en las que buscan la reconstrucción del empleo ${ }^{23}$, la perspectiva de género en los Objetivos de Desarrollo Sostenible $y$ el significado del término "empoderamiento" 24 , el gasto presupuestario de las políticas de género ${ }^{25}$, las políticas LGBTIQA $+{ }^{26}$ y las campañas públicas destinadas a crianzas no sexistas ${ }^{27}$, entre otras.

En ese contexto, la Junta Municipal del Distrito de Ciudad Lineal convocó una sesión ordinaria para el día 21 de enero a las 17:30 horas, incluyendo en el punto 8 de la Parte Resolutiva el tratamiento de una presentación el Grupo Municipal VOX, sintetizada de la siguiente manera:

Proposición no 2021/2744, presentada por el Grupo Municipal Vox, solicitando instar a la Junta Municipal de Distrito, y si no fuera de su competencia al Área competente, a cambiar el mural pictórico que se encuentra ubicado en la pared de la entrada principal del Centro Deportivo Municipal Concepción en la calle José del Hierro, por uno dedicado a deportistas y al deporte y que no contenga ningún mensaje político,únicamente deportivo ${ }^{28}$.

Otro dato contextual relevante es que el Grupo Municipal Socialista elevó para la misma sesión la Pregunta N²021/3558 para conocer las gestiones de la Junta en el período 2020-2021 en materia de retiro de simbología franquista y el cambio del nombre de las calles dedicadas a figuras dictatoriale, en conformidad con la Ley de Memoria

\footnotetext{
22 Congreso de los Diputados, Expte. 48633, 31 de julio de 2020.

${ }^{23}$ Congreso de los Diputados, Expte. 30078, 18 de mayo de 2020.

${ }^{24}$ Congreso de los Diputados, Expte. 18469, 26 de marzo de 2020.

25 Congreso de los Diputados, Comparecencia de la Sra. Ministra de Igualdad, 7 de octubre de 2020.

${ }^{26}$ Congreso de los Diputados, Comparecencia de la Sra. Ministra de Igualdad, 7 de octubre de 2020.

27 Congreso de los Diputados, Comparecencia de la Sra. Ministra de Igualdad, 7 de octubre de 2020.

28 Junta Municipal del Distrito de Ciudad Lineal, Orden del Día de la sesión ordinaria convocada para el día 21 de enero a las 17:30 horas [Publicada el 18 de enero de 2021].
} 
Histórica29. La lectura en la clave propuesta nos permite dar cuenta, prima facie, de las disímiles agendas que ambos grupos tienen en la materia: estrategias contra la "dictadura progre" del "feminismo radical" que busca hegemonizar las calles ${ }^{30}$, por un lado, y acciones progresistas de repudio al franquismo y memoria activa, por el otro.

El día 21 de enero de 2021 se aprueba la proposición de VOX por mayoría, siendo el resultado de la votación: 5 votos en contra del Grupo Municipal Más Madrid, 4 votos a favor del Grupo Municipal Partido Popular, 3 votos a favor del Grupo Municipal CiudadanosPartido de la Ciudadanía, 2 votos en contra del Grupo Municipal Socialista y el voto a favor del Grupo Municipal Vox ${ }^{31}$. Desde este momento, hasta el Pleno del Ayuntamiento desarrollado el día 26 de enero de 2021 se despliegan fuertes campañas a favor y en contra de la decisión tanto en redes sociales como en las calles ${ }^{32}$.

Respecto del mural cabe mencionar la importancia en la participación comunitaria tanto en su creación como posterior defensa, en la entrevista que se realizara sobre el caso en concreto, la informante hizo especial hincapié en esta circunstancia:

"(...) desde la Mesa de Igualdad de Ciudad Lineal de los Foros Locales del Ayuntamiento de Madrid, se propusiera un mural en el que se visibilizaran mujeres de la historia. Fue un proyecto en el que tanto la decisión de qué retratos de mujeres se pintarían, como la propia realización del mural se realizó de manera colectiva. El trabajo se adjudicó a una asociación que abrió el proceso de pintar a la participaron de colegios, institutos y vecinas y vecinos. Este proceso colectivo conllevó que la gente del barrio lo sintiera como propio. Cuando se anuncia que en el pleno del distrito se ha aprobado su eliminación, la ciudadanía salta frente a la barbarie, y el mural se convierte en un símbolo de la reivindicación de una sociedad más igualitaria. A pesar de las restricciones por la pandemia de coronavirus, la gente se echa a la calle a pedir que "El mural se queda". Tras un año de apenas contacto social, el mural se convierte además en un lugar de encuentro (...) La moción aprobada de eliminar el mural en una

\footnotetext{
29 Junta Municipal del Distrito de Ciudad Lineal, Orden del Día de la sesión ordinaria convocada para el día 21 de enero a las 17:30 horas [Publicada el 18 de enero de 2021].

30 Antonio Álvarez-Benavides y Francisco Jiménez Aguilar, 'Estrategias de comunicación de la nueva extrema derecha española. De Hogar Social a VOX, del alter-activismo a la doctrina del shock' (2020) 1(2) Estudios de la Paz y el Conflicto. Revista Latinoamericana 55.

31 Junta Municipal del Distrito de Ciudad Lineal, Resumen de Acuerdos de la sesión ordinaria del día 21 de enero de 2021.

32 Diario Público, 'Directo: Concentración contra el borrado de $\mathrm{n}$ mural feminista en el barrio de la Concepción de Madrid. [24 de enero de 2021]. Diario Público. En: $<$ https://www.publico.es/sociedad/mural-feminista-barrio-concepcion- 
Junta Municipal de Distrito fue revocada por el Pleno General del Ayuntamiento de Madrid a iniciativa del grupo municipal Más Madrid. La presión vecinal y su repercusión fue fundamental para que los partidos políticos se repensaran su voto" (Entrevista de fecha 10 de agosto de 2021).

El día 26 de enero de 2021 ocurren dos hechos políticos clave. Por la mañana, el alcalde decide eliminar del organigrama del Ayuntamiento la Dirección General de Igualdad asegurando que esa decisión "en ningún caso (...) es una cesión a Vox"33. En la tarde, se trata en el Pleno del Ayuntamiento de Madrid la moción de urgencia N²021/8000120 presentada por el Grupo Municipal Más Madrid para la preservación y mantenimiento de las acciones desarrolladas dentro del programa "Compartiendo Muros", especialmente en el Distrito de Ciudad Lineal ${ }^{34}$. El acuerdo adoptado aprueba la totalidad de la expresión de motivos de la moción presentada, sosteniéndose en el atentado que la eliminación del mural implicaría respecto de la legislación en materia de equidad de género. En relación al enfoque adoptado en este trabajo, interesa resaltar que el acuerdo señala:

(...) el reconocimiento del papel de las mujeres, y su relevancia social es un eje fundamental de estas políticas y el mural contribuye a ello, en cuanto pone en valor una serie de mujeres que han ejercido influencia relevante en el ámbito literario, artístico, deportivo, político, científico y político ${ }^{35}$.

Se pone en juego en esta declaración no sólo la relación entre equidad, memoria y "puesta en valor" del rol histórico de las mujeres ${ }^{36}$, sino también la importancia del arte para la memoria ${ }^{37}$, cuestiones que también quedaron plasmadas en el debate del Pleno. En efecto, el día 26 de enero la cuestión del mural fue llevada a una gran cantidad de tópicos a resolverse en esa sesión, antes del tratamiento de la moción de urgencia. Por ejemplo, al momento de tratar la proposición sobre la implementación de mecanismos de participación ciudadana respecto del catálogo de elementos protegidos dentro del Plan General de

33 Diario Público Agencias, 'Almeida elimina la Dirección General de Igualdad del Ayuntamiento de Madrid' [26 de enero de 2021]. Diario Público. En: https://publico.es/politica/almeida-elimina-direccion-general-igualdad-

ayuntamiento-madrid.html

34 Pleno del Ayuntamiento de Madrid, Acuerdos adoptados en la sesión 1/2021.

35 Pleno del Ayuntamiento de Madrid, Acuerdos adoptados en la sesión 1/2021, 30.

${ }^{36}$ Lelya Elena Troncoso Pérez \& Isabel Piper Shafir, 'Género y memoria: articulaciones críticas feministas' (2015 15 Revista Athenea Digital.

${ }^{37}$ Marianne Hirsch, 'Introduction: Practicing Feminism, Practicing Memory', en Ayşe Gül Altınay and María José Contreras, Marianne Hirsch, Jean Howard, Banu Karaca \& Alisa Solomon(Eds..) Women Mobilizing Memory (Columbia University Press 2019). 
Integración Urbana, el edil Miguel Montejo Bombín, del Grupo Municipal Más Madrid, señaló que "proteger el patrimonio cultural es una forma de proteger no el pasado sino nuestro futuro" y que la realización del catálogo pretendido debía ser desde "este entendimiento del patrimonio cultural como soporte de nuestra identidad y de nuestra memoria colectiva" 38 .

A su turno, el portavoz del Grupo Municipal Socialista, José Vicente Hernández Fernández, se manifestó al tratar la Proposición de reconocimiento a los involucrados en las tareas vinculadas a la tormenta "Filomena", dirigiéndose directamente a la vicealcaldesa Villacín:

Permítame que hablando de homenajes, termine hablando de un mural que ustedes quieren borrar, señora Villacís, en una nueva vergonzante concesión a VOX, otra más. Ese mural es también un homenaje en sí mismo a todas las mujeres con sus ideas y su ejemplo personal. Permitieron a nuestra sociedad avanzar en derechos y libertades. Por favor, reflexionen en la imagen que está dando Madrid en el mundo (... $)^{39}$

Al momento del tratamiento de la moción de urgencia propiamente, la concejala de VOX, Arántzazu Cabello, arremetió fuertemente no sólo contra la "ideología de género" y las mujeres reconocidas por llevar un fusil en la mano o haber estado alentando revoluciones" 40 , sino también contra la portavoz de Ciudadanos "que no está siendo leal a un concejal de su propia adscripción"41, pues ese Grupo Municipal decidió votar a favor la moción de urgencia en contradicción con su participación en la Junta del Distrito. Esto último tuvo una fuerte repercusión en redes sociales, donde las cuentas oficiales de VOX desplegaron con fuerza su épica belicista y descalificatoria característica ${ }^{42}$.

Enma López Araujo del Grupo Municipal Socialista acusó directamente al gobierno local abiertamente: "son ustedes un gobierno roto, un gobierno roto que quiere borrar la memoria de Rosa Parks, de Valentina Tereshkova, que quieren borrar la memoria de Frida Khalo,

\footnotetext{
38 Pleno del Ayuntamiento de Madrid, Diario de Sesiones, Sesión ordinaria del 26 de enero de 2021 [Publicado el 8 de febrero de 2021], 27.

39 Pleno del Ayuntamiento de Madrid, Diario de Sesiones, Sesión ordinaria del 26 de enero de 2021 [Publicado el 8 de febrero de 2021], 52-53.

40 Pleno del Ayuntamiento de Madrid, Diario de Sesiones, Sesión ordinaria del 26 de enero de 2021 [Publicado el 8 de febrero de 2021], 97.

41 Pleno del Ayuntamiento de Madrid, Diario de Sesiones, Sesión ordinaria del 26 de enero de 2021 [Publicado el 8 de febrero de 2021], 97.

42 Monforte, Marta. 'PP y Vox critican la 'deslealtad' de Cs por querer mantener el mural feminista cinco días después de apoyar su retirada'. [26 de enero de 2021] Diario Público. En: <https://publico.es/politica/mural-feminista-ciudad-lineal-ppvox-critican-deslealtad-cs-mantener-mural-feminista-cinco-dias-despues-apoyarretirada.html>
} 
de Rigoberta Menchú..."43, a lo que la vicealcaldesa respondió, entre otras cosas:

(...) me pregunto por qué no están otras mujeres también abanderadas del feminismo que han defendido otra ideología, por qué no está por ejemplo Margaret Thatcher, por qué no está por ejemplo Concepción Arenal, por qué no está por ejemplo santa Teresa de Jesús si ustedes quieren, todas esas mujeres que fueron abanderadas; pero no, solo están aquellas que defienden el feminismo y que además son de izquierdas. ${ }^{44}$

Esto último tuvo fuertes repercusiones en redes sociales donde, desde diferentes cuentas, usuarios y usuarias citaban frases antifeministas de Margaret Thatcher y compartían memes y otras formas de humor virtuales ${ }^{45}$, pero pinta de cuerpo entero la magnitud de la disputa: ¿qué, quién y cómo inscribe la memoria?

La exposición la cerró la secretaria del Grupo Municipal Más Madrid, Carolina Pulido Castro, quien intervino reforzando los argumentos sostenidos desde la decisión del 21 de enero ${ }^{46}$ y haciendo fuerte hincapié en la movilización popular ${ }^{47}$ que impedirá que el gobierno borre a las mujeres de la historia y que no permitirá "ningún cambio en el mural, porque el mural se queda, el mural no se toca y serán mil murales; que, por cierto, también es patrimonio cultural"48

Tras las acciones iniciadas por Más Madrid, las movilizaciones vecinales en defensa del mural y el apoyo del resto de los partidos de izquierda con presencia en el Ayuntamiento, es que el 10 de junio se decidió en el Ayuntamiento su continuación, el 16 de junio el Colegio Honduras de Vallecas rechazó la moción de VOX para remover el mural y el 25 de agosto el Ayuntamiento ordenó su restauración tras la vandalización sufrida en la madrugada del $8 \mathrm{M}$ aunque vetó en la

\footnotetext{
43 Pleno del Ayuntamiento de Madrid, Diario de Sesiones, Sesión ordinaria del 26 de enero de 2021 [Publicado el 8 de febrero de 2021], 98.

${ }^{44}$ Pleno del Ayuntamiento de Madrid, Diario de Sesiones, Sesión ordinaria del 26 de enero de 2021 [Publicado el 8 de febrero de 2021], 98.

${ }^{45}$ Tremending, 'De los creadores de 'feminismo liberal' llega la Thatcher feminista: cachondeo con lo que ha dicho Villacís sobre el mural de Ciudad Lineal' [ 29 de enero de 2021]. Diario público. En: <https://publico.es/tremending/2021/01/28/de-loscreadores-de-feminismo-liberal-llega-la-thatcher-feminista-cachondeo-con-lo-que-

ha-dicho-villacis-sobre-el-mural-de-ciudad-lineal/>

${ }^{46}$ Europapress, 'Más Madrid tacha de 'títeres de los trumpistas' a Ayuso y Almeida'. [24 de enero de 2021]. Diario Público. En: <https://publico.es/videos/933713/masmadrid-tacha-de-titeres-de-los-trumpistas-a-ayuso-y-almeida>

47 Europapress, 'Una concentración ciudadana pide el mantenimiento del mural de La Concepción' [24 de enero de 2021]. Diario Público. En: $<$ https://publico.es/videos/933734/una-concentracion-ciudadana-pide-elmantenimiento-del-mural-de-la-concepcion>

48 Pleno del Ayuntamiento de Madrid, Diario de Sesiones, Sesión ordinaria del 26 de enero de 2021 [Publicado el 8 de febrero de 2021], 99.
} 
decisión la participación ciudadana en el proceso solicitada por los artistas en una cláusula contractual. Sobre esto último, la informante comentó que: "la lucha continúa, porque a pesar de haberse aprobado que el Mural se queda, no fue protegido del vandalismo de grupos de extrema derecha, y aún ahora seguimos demandando que se restaure" (Entrevista de fecha 10 de agosto de 2021).

\section{CONCLUSIONES}

La intersección entre los campos de estudio abordados en este trabajo -género y memoria- permite una mirada crítica e innovadora con posibilidad de dar respuesta al actual avance de las derechas, en tanto reivindica las luchas por los derechos humanos en contextos de violencia estatal $y$, en particular aquellas relacionadas con el feminismo, en las que la movilización de la memoria como política activa contrahegemónica hace frente a los movimiento reaccionarios y neoconservadores. A su vez, rescata la importancia de las mujeres en la producción de conocimiento y su participación en el espacio público del cual han sido relegadas históricamente desde una visión esencialista que les impidió inscribir sus memorias.

El caso del mural feminista de Ciudad Lineal reabre el debate: ¿qué, quién y cómo se inscribe la memoria en la ciudad? En un contexto de exclusión de las mujeres del acceso y goce del derecho a la ciudad, el discurso instaurado por el partido VOX con estrategias contra la "dictadura progre" del "feminismo radical" buscó hegemonizar las calles, por un lado, y promovió acciones progresistas de repudio al franquismo y memoria activa, por el otro. Es así que, la disputa por el mural fue mucho más allá de una decisión estética o urbanística en sentido estricto, sino que en ella subyace la lucha por la toma de decisiones en la ciudad en general, y por quiénes logran inscribir la memoria en el espacio en particular. La escasez de espacios de la memoria que satisfagan los estándares internacionales en la materia perpetúa las inscripciones que invisibilizan a las víctimas del franquismo y antecede y fundamenta la exclusión de las mujeres del espacio público.

Finalmente, cabe mencionar que la disputa trascendió las fronteras no sólo del distrito sino también de Madrid y España, en una clara muestra de cómo la apropiación comunitaria de los espacios públicos y la inscripción de las memorias de las mujeres resultan ser procesos democratizadores en las ciudades que abren nuevas líneas de discusión en torno a los Derechos Humanos en general con perspectiva de género en su especificidad. Para ello, se contó con una gran difusión en las redes y en los medios de comunicación el conflicto y se logró que "un mural que solo era conocido por las personas de un barrio, se convierta en un símbolo internacional. Recibimos cartas de solidaridad de todo el mundo en defensa del mural, fuimos noticia en telediarios de televisiones de varios países, y el mural se replicó en muchos 
lugares, tras difundir las plantillas. "Si no quieren uno, tendrán

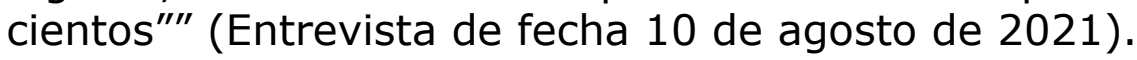

\section{BIBLIOGRAFÍA}

Aladro, A. 'Capitalismo, patriarcado y ciudad: generalidades de la producción de lo urbano desde una perspectiva de género' [2019] 21 Revista Derecho y Ciencias Sociales.

Aladro A, 'El acceso a la ciudad de las mujeres' [2020], II Coloquio Internacional de Conflictos Urbanos, Jaén de la Frontera, Universidad de Cádiz.

Balinhas D, 'Populismo y nacionalismo en la "nueva" derecha radical española' [2020] 13 Pensamiento al margen. Revista Digital de Ideas Políticas 69.

Bacci, C. ' 'Ahora que estamos juntas: memorias, políticas y emociones feministas' [2020] 28 Revista Estudos Feministas 1.

Borja J, Revolución urbana y derechos ciudadanos (Editorial Café de las Ciudades 2014).

Celiberti, L.E, 'Desatar, desnudar... reanudar' [2015] 20 Revista Estudos de Sociologia, Araraquara 291.

Ferrer González, M.C, 'Memoria histórica y democrática con perspectiva de género' [2019]. 96 Revista PH.

Hirsch, M. 'Introduction: Practicing Feminism, Practicing Memory', en Ayşe Gül Altınay and María José Contreras, Marianne Hirsch, Jean Howard, Banu Karaca \& Alisa Solomon(Eds..) Women Mobilizing Memory (Columbia University Press 2019).

Jelin, E. \& Sutton, B., 'Memoria, género y activismo. Resistencia a la dictadura y lucha por el aborto legal' [2021] 11(22) Aletheia.

Larreche, J. I., 'Lo barrial del barrio. Una discusión desde el habitar subalterno en Bahía Blanca (Argentina)' [2019] 15(2) Geograficando 58.

Till K, 'Wounded cities: Memory-work and a place-based ethics of care' [2012] 31 Revista Political Geography 1.

Troncoso Pérez, L.E \& Piper Shafir, I., 'Género y memoria: articulaciones críticas feministas' [2015]. Revista Athenea Digital, vol.15, núm.1, pp.65-90.

Vasilachis de Gialdino I, Discurso Político y Prensa escrita. Un análisis sociológico, jurídico y lingüístico (Gedisa 1997).

Vasilachis De Gialdino I (Coord.), Estrategias de investigación cualitativa (Editorial Gedisa 2006).

\section{1.- Documentos}

Congreso de los Diputados, Expte. 18469, 26 de marzo de 2020.

Congreso de los Diputados, Expte. 30078, 18 de mayo de 2020.

Congreso de los Diputados, Expte. 48633, 31 de julio de 2020. 
Congreso de los Diputados, Comparecencia de la Sra. Ministra de Igualdad, 7 de octubre de 2020.

Junta Municipal del Distrito de Ciudad Lineal, Orden del Día de la sesión ordinaria convocada para el día 21 de enero de 2021 a las 17:30 hs. [Publicada el 18 de enero de 2021]

Junta Municipal del Distrito de Ciudad Lineal, Resumen de Acuerdos de la sesión ordinaria del día 21 de enero de 2021.

Pleno del Ayuntamiento de Madrid, Acuerdos adoptados en la sesión $1 / 2021$.

Pleno del Ayuntamiento de Madrid, Diario de Sesiones, Sesión ordinaria del 26 de enero de 2021 [Publicado el 8 de febrero de 2021]

\section{2.- Material periodístico}

Diario Público, 'Directo: Concentración conra el borrado de $n$ mural feminista en el barrio de la Concepción de Madrid. [24 de enero de 2021]. Diario Público. En:

<https://www.publico.es/sociedad/mural-feminista-barrioconcepcion-madrid.html>

Diario Público Agencias, 'Almeida elimina la Dirección General de Igualdad del Ayuntamiento de Madrid' [26 de enero de 2021]. Diario Público. En: <https://publico.es/politica/almeida-eliminadireccion-general-igualdad-ayuntamiento-madrid.html>

Europapress, 'Una concentración ciudadana pide el mantenimiento del mural de La Concepción' [24 de enero de 2021]. Diario Público. En: <https://publico.es/videos/933734/una-concentracionciudadana-pide-el-mantenimiento-del-mural-de-la-concepcion>

Europapress, 'Más Madrid tacha de 'títeres de los trumpistas' a Ayuso y Almeida'. [24 de enero de 2021]. Diario Público. En: <https://publico.es/videos/933713/mas-madrid-tacha-de-titeres-delos-trumpistas-a-ayuso-y-almeida $>$

Marta Montoforte, 'Mural feminista: La presión política y vecinal obliga al Ayuntamiento de Madrid a recular: el mural feminista se mantendrá' [26 de enero de 2021]. En: Diario Público. Disponible en: <https://www.publico.es/politica/mural-feminista-presionpolitica-vecinal-obliga-ayuntamiento-madrid-recular-muralfeminista-mantendra.html>

Monforte, Marta. 'PP y Vox critican la 'deslealtad' de Cs por querer mantener el mural feminsta cinco días después de apoyar su retirada'. [26 de enero de 2021] Diario Público. En: $<$ https://publico.es/politica/mural-feminista-ciudad-lineal-ppvox-critican-deslealtad-cs-mantener-mural-feminista-cincodias-despues-apoyar-retirada.html> 